\title{
Alternative approach to variable wavelength interferometry
}

\author{
Dariusz Litwin, ${ }^{*}$ Kamil Radziak, Jacek Galas \\ Eukasiewicz Research Network-Tele- and Radio Research Institute, Centre for Applied Photonics, 11 Ratuszowa St., \\ 03-450 Warsaw, Poland
}

Received November 14, 2020; accepted December 22, 2020; published December 31, 2020

\begin{abstract}
The paper presents an alternative technique of calculating the retardance of quartz waveplates. The technique utilizes a continuously tuned wavelength, which identifies the zero-order fringe and simultaneously facilitates high repeatability of an optical path difference across the entire visible spectrum. Unlike in a classical method, precise monitoring of a current increase or decrease of interference order is not required. The discussion includes a comparison of the standard deviation between classical and novel approaches.
\end{abstract}

Variable wavelength interferometry (VAWI) is a useful optical technology in characterising microscopical objects. The family of these methods with the use of the Wollaston prism-based interferometer has been proposed originally by Pluta in the 1980s [1-4]. These techniques for transmitted and reflected light are suitable for measuring objects that create an optical path difference (OPD) greater than one wavelength. The methodology consisting in measuring a simple deflection of interference fringes is not supportive because of the lack of integrity of interference fringes. VAWI can be applicable in an industrial environment for measuring waveplate retardance [5], textile and optical fibers, and strips of thin films [6-8]. A detailed description of the original approach is beyond the scope of this paper but can be found in the cited publications. The technique consists in monitoring and measuring the change of phase in the fringe field during the continuously varying wavelength of a light source. In the early systems all operations and following calculations had to be performed manually, which was a tedious and days-long procedurenot very attractive from the commercial point of view. The researcher had to watch the moving fringe field and identify selected, easy-to-recognize phase values i.e. the minima and maxima of fringes and write down the corresponding wavelengths for further calculations. In the most known VAWI methodology these characteristic locations were called 'coincidences' since they referred to equal phases of the fringe pattern in the empty field and in the object region. In other VAWI methods these coincidences occur between specially selected reference lines and fringes. In automated systems coincidences have been replaced by more efficient registration of phases for subsequent corresponding wavelengths.

\footnotetext{
*E-mail: dariusz.litwin@itr.lukasiewicz.gov.pl; adlitwin@pro.onet.pl
}

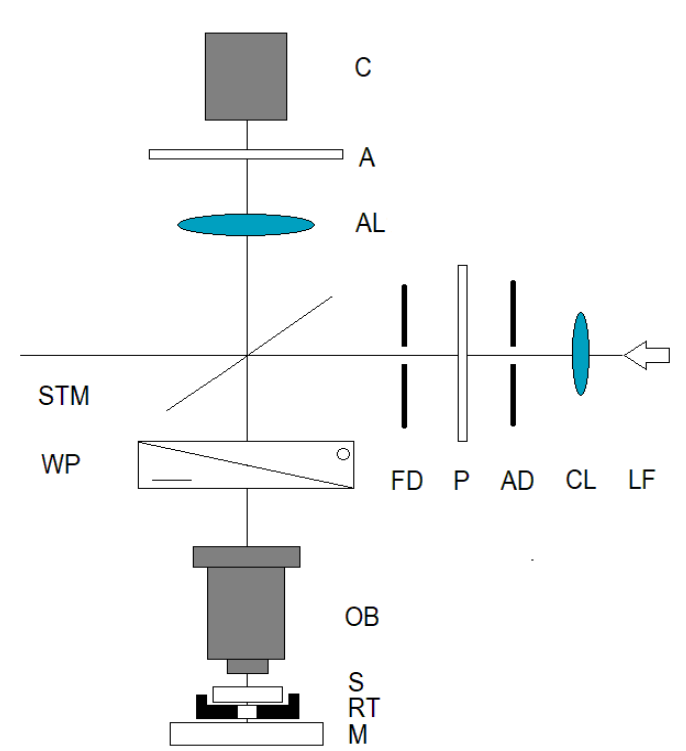

Fig.1. Optical setup of the VAWI interferometer in reflected-light mode.

We built a new version of the system arranged in reflected-light mode for industrial laboratories of optical factories [9]. The instrument was configured to be used for measuring the retardance of waveplates [11], which is necessary in the production process quality control (Fig. 1). The advantage of the instrument is that the light passes through the measured plate twice and the interfringe distance is twice smaller compared to the transmitted-light system, which together creates potential for higher accuracy. The setup of the new microinterferometeric systems is similar compared to its first prototypes [9]. The system includes a microscopic objective $\mathrm{OB}$ of magnification that equals $10 \times$ or $20 \times$, and the Köhler illumination system (LF-lamp filament). The illumination system utilizes the Czerny-Turner monochromator as a variable monochromatic light source (not showed in the figure). The other components are: CL-Collector Lens, FD-field diaphragm, P-polarizer, Aanalyser, M-mirror, STM-semitransparent mirror, ADaperture diaphragm, S-specimen (a retarder), WPWollaston prism, and AL -auxiliary lens. The interference field of straight parallel fringes is produced by a classical Wollaston prism WP placed between a crossed polarizer and analyzer, which results in a dark zero-order fringe. 
The light propagates through the Wollaston prism twice in a reflected-light system. The microscope objective, apart from its typical function, performs as a condenser. The Wollaston prism acts like a compensator of spatially variable optical path difference between ordinary and extraordinary beams. Thanks to the system's selfcompensating feature a large numerical aperture of the illuminator can be realized [1]. The microinterferometer has been configured for measuring waveplates. The retarders $S$ are placed under the objectives at the rotation table RT so that they can be measured at their two optical axes orientations, at $90^{\circ}$ angular distance. The entire field of view is covered by the retarder in question.

As the VAWI theory has been already explained in many previous papers [1-8], we will recall some background information. Waveplates produce the phase retardation $\varphi$ and optical path deference OPD, respectively:

$$
\varphi=\frac{2 \pi}{\lambda}\left(n_{e}-n_{o}\right) 2 g, \quad O P D=\left(n_{e}-n_{o}\right) 2 g,
$$

where $n_{o}$, and $n_{e}$ are the refractive indices: ordinary and extraordinary, respectively, $g$ is the thickness of the plate, which is doubled in the reflected-light configuration. We assume that we measure crystal quartz waveplates in the form of a single plane-parallel plate i.e. a multiple order waveplate. When a waveplate is placed above the mirror it introduces a certain shift of the zero-order fringe. The corresponding OPD (in nanometers) can be expressed as:

$$
m_{1} \lambda_{1}=2 g\left(n_{e 1}-n_{o 1}\right) \text {. }
$$

When the wavelength is decreased from $\lambda_{1}$ to $\lambda_{s}$, the process moves from the red region to blue (' $\mathrm{s}$ ' means 'subsequent') and the equation evolves to the form:

$$
\left(m_{1}+q_{s}\right) \lambda_{s}=2 g\left(n_{e s}-n_{o s}\right)
$$

Variable $m_{1}$ is called the initial interference order, $\left(m_{l}+q_{s}\right)$ is the current interference order, and $q_{s}$ is the increment or decrement of the current interference order. After dividing the sides of the Eqs. (2) and (3) and utilizing the interfringe distances $b_{1}, b_{s}$ instead of wavelengths [1, 12], we receive a simpler equation though on a strict assumption that the sample is made of quartz:

$$
m_{1}=\frac{b_{s} q_{s}}{b_{1}-b_{s}}, \quad O P D\left(\lambda_{s}\right)=\left(m_{1}+q_{s}\right) .
$$

The last equations indicate that to calculate the initial interference order and then OPD of waveplates, it is necessary to measure only the period of fringes and current interference order ' $q_{s}$ '. The required quantities can be collected only by means of image processing. In order to display the user-oriented characteristics i.e. retardance or OPD vs wavelengths, it is necessary to calibrate the system with the use of a collection of stable laser sources.
The new approach is entirely numerical and based on an image processing concept. The beginning is exactly the same as in the classical approach. We collect a fringe image sequence for subsequent wavelengths. We extract from each fringe image an averaged line, and fit a sin function. Thanks to this operation we have a collection of sinusoids (Fig. 2), each of which refers to a different subsequent wavelength. In the previous process we would use the initial sinusoid phase to calculate $\mathrm{q}$ and follow the above equations, i.e. Eqs. $(3,4)$. Here, however, in the next step, the image is converted into a set of straight lines [10] that are fitted to the minima and maxima of fringes (Fig.3). In the final step it is enough to find the line which is inclined at the angle closest to the right angle and simultaneously refers to the dark fringe (minimum). This is the position of our zero-order fringe.

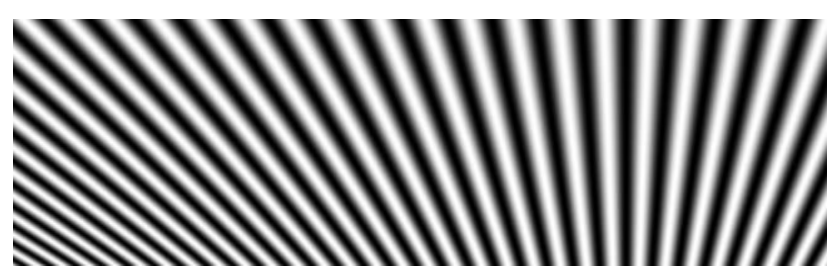

Fig. 2. The image of a collection of sinusoids in the virtual space.

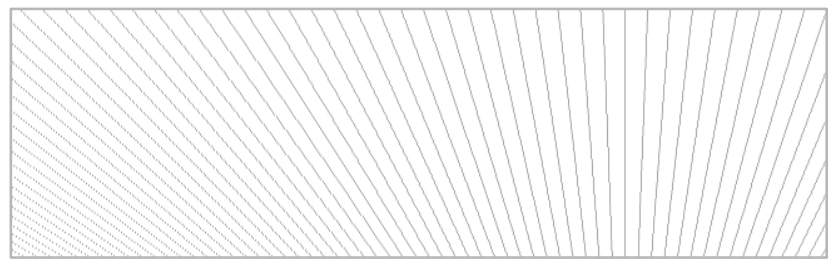

Fig. 3. The image of minima and maxima of sinusoids

Since both the periods of fringes and the initial phases of sinusoids suffer from measurement errors, the process is repeated iteratively. In each iteration, the periods are averaged according to the spaces between the fitted lines and then the sinusoids are shifted so that their minima coincide with the position of the zero order line. The latter should be, in these circumstances, vertical [10]. Three iterations produce excellent images (Figs. 2 and 3). Having two coordinates of the zero-order fringe found for the mentioned two angular orientations of the retarder i.e. $a_{0}$ and $a_{90}$ and sequence of periods of the fringes $b\left(\lambda_{\mathrm{s}}\right)$, it is possible to calculate the OPD function across the whole spectrum:

$$
O P D\left(\lambda_{s}\right)=\frac{a_{0}+a_{90}}{4 b\left(\lambda_{s}\right)} .
$$

Clearly, in the real image produced in the interferometer we cannot see the zero-order fringe but only a small part of highly inclined fringes. The whole process is then performed in the virtual space of sinusoids that are created analytically on fitting parameters, i.e. the initial phase shifts and periods. 
Our comparative studies are based on a single set of fringe images collected at one measurement session (consisted of 25 meaurements) of the same quartz waveplate. In Fig. 4 we compare $O P D s$. The curves are almost identical showing a slight difference in the blue region.

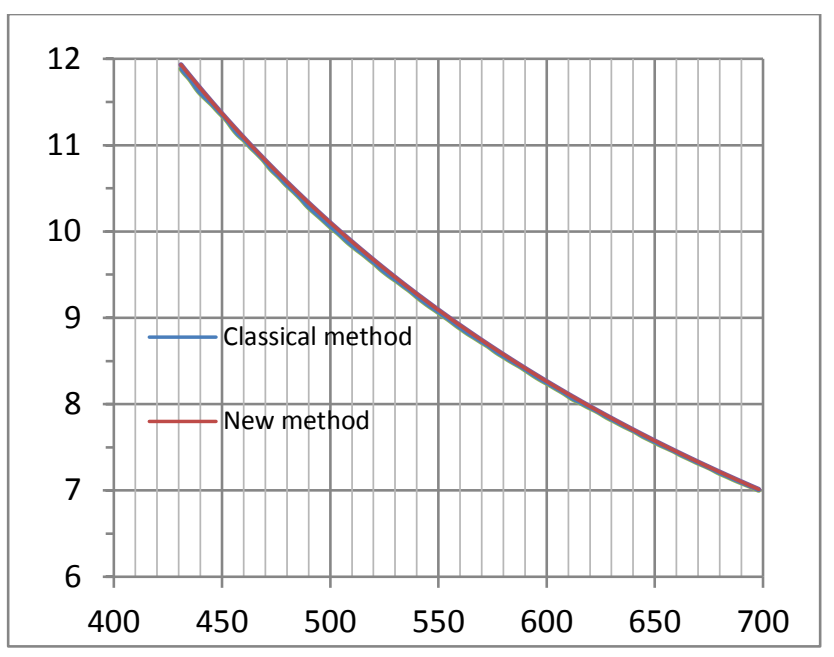

Fig. 4. Optical path difference $[\lambda]$ vs. wavelength $[\mathrm{nm}]$.

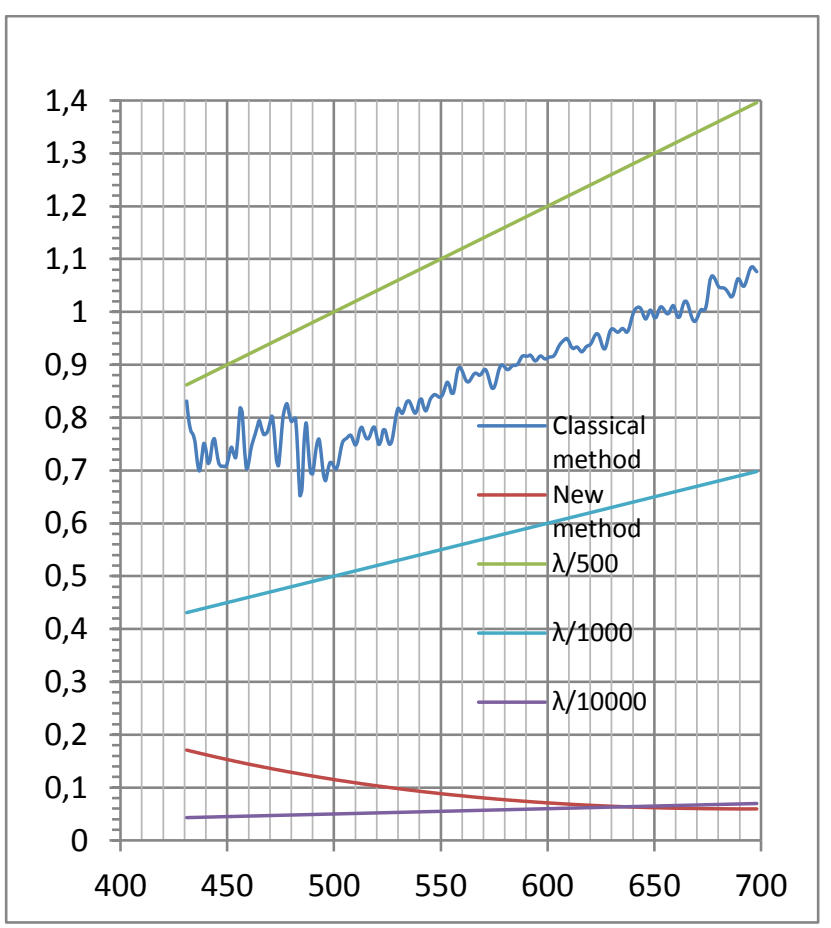

Fig. 5. Standard deviation $[\mathrm{nm}]$ vs wavelength $[\mathrm{nm}]$.

This identity verifies mutually both approaches experimentally. In Fig. 5 we compare the standard deviations $S_{d}$ calculated for 25 measurements. In the case of the classical methods, $S_{d}$ is rough demonstrating significant deterioration in the blue region where the fringes are of the lowest contrast (white LED has been used). An averaged $S_{d}$ level is placed between $\lambda / 500$ and $\lambda / 1000$, which might be regarded as satisfactory. The roughness is caused by the measurand $q$, which suffers from measuring errors, especially in the blue region. Fortunately, in the case of the new method, thanks to the global fitting of the sinusoid collection to the multiwavelength fringe field, $S_{d}$ is smooth and extremely low.

In conclusions, we have demonstrated an alternative technique for measuring the retardance of quartz waveplates. The approach has turned out to be very promising. It offers much higher repeatability across the whole visible spectrum compared to the classical VAWI methodology. Although the approach is not the only one possible, it produces excellent results using a relatively simple (meaning fast) algorithm which, when combined with one-shot interference architecture [12], offers quick characterization of the waveplate within a few-second timeframe.

The VAWI project was partly funded by the Polish Ministry of Education and Science, grant no. 4T07D 00527.

The Centre for Applied Photonics is a part of the Łukasiewicz Research Network-Tele- and Radio Research Institute (Ł-ITR). It was created in 2020 after incorporating the Institute of Applied Optics-INOS into L-ITR and continues the line of research and tradition of the former. The newly established Łukasiewicz Research Network is organized in a way similar to FraunhoferGesellschaft.

\section{References}

[1] M. Pluta, Advanced Light Microscopy (Vol. 3, PWN, Elsevier, Warszawa-Amsterdam-London-New York-Tokyo, 1993).

[2] M. Pluta, J. Opt. Soc. Am. A 4(11), 2107 (1987).

[3] M. Pluta, J. Microscopy 149(2), 97 (1988).

[4] M. Pluta, J. Microscopy 145(2), 191 (1987).

[5] M. Pluta, Opt. Laser Technol. 19(3), 131 (1987).

[6] D. Litwin, A.M. Sadik, Opt. Applicata 28(2), 139 (1998).

[7] A. Sadik, W.A. Ramadan, D. Litwin, Meas. Scien. Technol., IOP Publishing 14(10), 1753 (2003).

[8] J. Galas, S. Sitarek, D. Litwin, M. Daszkiewicz, Proc. SPIE 10445 1044504 (2017).

[9] D. Litwin, J. Galas, N. Błocki, Proc. SPIE 6188, 61880F (2006).

[10] J. Galas, D. Litwin, M. Daszkiewicz, Proc. SPIE 10142, 101421R (2016).

[11] D. Litwin, J. Galas, M. Daszkiewicz, T. Kryszczyński, A. Czyżewski, K. Radziak, Phot. Lett. Poland 11(2), 29 (2019). https://doi.org/10.4302/plp.v11i2.898

[12] D. Litwin, K. Radziak, J. Galas, Proc. SPIE 11581, 115810O, (2020). https://doi.org/10.1117/12.2580386 